\title{
Identification and molecular characterisation of a homozygous missense mutation in the ADAMTS10 gene in a patient with Weill-Marchesani syndrome
}

\author{
Hannes Steinkellner ${ }^{1,3}$, Julia Etzler ${ }^{1,3}$, Laura Gogoll ${ }^{1}$, Jürgen Neesen ${ }^{1}$, Eva Stifter ${ }^{2}$, Oliver Brandau ${ }^{1}$ \\ and Franco Laccone ${ }^{\star}, 1$
}

Weill-Marchesani syndrome is a rare disorder of the connective tissue. Functional variants in ADAMTS10 are associated with Weill-Marchesani syndrome-1. We identified a homozygous missense mutation, c.41T $>$ A, of the ADAMTS10 gene in a 19-yearold female with typical symptoms of WMS1: proportionate short stature, brachydactyly, joint stiffness, and microspherophakia. The ADAMTS10 missense mutation was analysed in silico, with conflicting results as to its effects on protein function, but it was predicted to affect the leader sequence. Molecular characterisation in HEK293 Ebna cells revealed an intracellular mis-targeting of the ADAMTS10 protein with a reduced concentration of the polypeptide in the endoplasmic reticulum. A large reduction in glycosylation of the cytoplasmic fraction of the mutant ADAMTS10 protein versus the wild-type protein and a lack of secretion of the mutant protein are also evident in our results. In conclusion, we identified a novel missense mutation of the ADAMTS10 gene and confirmed the functional consequences suggested by the in silico analysis by conducting molecular studies.

European Journal of Human Genetics (2015) 23, 1186-1191; doi:10.1038/ejhg.2014.264; published online 3 December 2014

\section{INTRODUCTION}

Weill-Marchesani syndrome (WMS) is a very rare disorder of the connective tissue. It is characterised by the presence of symptoms in several structures, such as the skeleton (proportionate short stature, joint stiffness, brachydactyly, scoliosis, lumbar lordosis, and maxillary hypoplasia), the eye (microspherophakia, ectopia lentis, severe myopia, glaucoma, shallow anterior chamber, cataract, and blindness), and the cardiovascular system (aortic valve stenosis, pulmonary valve stenosis, mitral valve insufficiency, persistent ductus arteriosus, and ventricular septal defect). ${ }^{1-5}$ The disease is inherited mostly as an autosomal recessive trait due to variants of the ADAMTS10 gene. ${ }^{6,7}$ Recently, a family with autosomal recessive WMS3 has been described for the LTBP2 gene. ${ }^{8}$ Additionally, in-frame deletions of the FBN1 gene have been described in dominantly inherited cases of WMS2 $2^{9,10}$ and some families with WMS or WMS-like syndrome due to variants of the ADAMTS17 gene. ${ }^{11}$

To date, only three missense variants, ${ }^{11,12}$ two non-sense variants ${ }^{6}$ and two splice site variants ${ }^{6}$ have been reported for the ADAMTS10 gene. Here we report a new missense variant affecting the leader peptide of the ADAMTS10 protein and its functional characterisation in a patient with a classic form of WMS1.

\section{MATERIALS AND METHODS}

Sequence analysis of the ADAMTS10 gene

Mutation screening was performed by PCR amplification of all coding exons of the ADAMTS10 gene (NM_030957.2) and subsequent sequence analysis by Sanger sequencing. The identified variant was confirmed with an independent PCR and sequencing reaction for the patient's and her parents' DNA (Supplementary Figure S3). The identified variant and relevant patient information were submitted to the LOVD ADAMTS10 mutation database (http://grenada.lumc.nl/LOVD2/eye/home.php?select_db=ADAMTS10).

\section{Construction of wild-type and mutant ADAMTS10 expression plasmids}

The complete wild-type ADAMTS10 coding sequence was amplified from human fibroblast RNA by RT-PCR using the primers 5'-ATGGCTCCCGCCTG CCAGATCCTCC-3' and 5'-GGGTGCCGCGCGCCCCCTAGTGG- $3^{\prime}$. The PCR product was cloned into pcDNA 3.1(+)/Myc-His B (Invitrogen, Carlsbad, CA, USA) for the expression of full-length ADAMTS10 with C-terminal tandem Myc and His6 tags. For introduction of the mutation c.41T > A, 5'-ATG GCTCCCGCCTGCCAGATCCTCCGCTGGGCCCTCGCCCTGGGGCTGGGC $\mathrm{C}-3^{\prime}$ was used as the forward primer. Complete coding sequences of wild-type and mutant ADAMTS10 cDNAs were cloned into the pd2eGFP-N1 vector (BD Biosciences/Clontech, Heidelberg, Germany) for expression of both wild-type and mutant ADAMTS10-d2eGFP fusion proteins. All expression plasmids were sequence verified.

\section{In silico analysis of the ADAMTS10 c.41T $>$ A mutation}

In silico mutation prediction analysis was performed using Mutation Taster (http://www.mutationtaster.org), PolyPhen-2, ${ }^{13}$ and SIFT. ${ }^{14}$ Analysis of a potential change in the ADAMTS10 signal peptide properties was performed on the first $60 \mathrm{~N}$-terminal amino acids of the wild-type and mutant (c.41T >A) human ADAMTS10 proteins using the publicly available SignalP World Wide Web prediction server version 4.1 (http://www.cbs.dtu.dk/services/SignalP/). ${ }^{15}$ The method incorporates testing for cleavage sites and signalling function based on neural networks.

Cell culture and transient transfection

HEK 293 Ebna (HEK 293E) cells were maintained in Dulbecco's modified Eagle's medium supplemented with $10 \%$ foetal bovine serum (from PAA 
Laboratories, Pasching, Austria) and penicillin/streptomycin (Gibco/Life Technologies, Darmstadt, Germany). The cells were plated on 35-mm dishes $12 \mathrm{~h}$ before $4 \mu \mathrm{g}$ of plasmid DNA was added using jetPrime transfection reagent (Peqlab Biotechnologie, Erlangen, Germany) according to the manufacturer's protocol. After $6 \mathrm{~h}$ of incubation, the medium was removed, and the cells were cultured in fresh medium or serum-free medium (Pro293 CD serum-free medium from Lonza, Visp, Switzerland) for another $18-42 \mathrm{~h}$. The cells were then used for distribution studies or the deglycosylation assay.

\section{Expression and characterisation of ADAMTS10 and ADAMTS10_L14Q polypeptides}

Six hours after transient transfection of HEK 293E cells with ADAMTS10-MycHis6 or ADAMTS10_L14Q-Myc-His6 plasmid DNA, the medium was removed, and the cells were cultured in Pro293 CD serum-free medium for another $42 \mathrm{~h}$, when the cells reached confluence. The medium was collected and concentrated 10-fold using a centrifugal ultrafiltration concentrator (Centrisart I $20 \mathrm{kDa}$, Sartorius AG, Göttingen, Germany). The cells were harvested and lysed in ice-cold $1 \times$ lysis reagent (Promega, Heidelberg, Germany) with $1 \times$ protease inhibitor cocktail (no. P8340) and $1 \mathrm{~mm}$ phenylmethyl sulphonylfluoride (both from Sigma-Aldrich, St Louis, MO, USA). After a 20 -min incubation on ice, the cell lysates were centrifuged at $10000 \mathrm{~g}$ for $10 \mathrm{~min}$ at $4{ }^{\circ} \mathrm{C}$. The supernatant was collected, and the protein concentration was determined by a modified Bradford assay (Bio-Rad, Vienna, Austria). Western blotting with anti-His6 polyclonal antibody (Cell Signaling Technology, Danvers, MA, USA) was used to determine the expression of ADAMTS10 and ADAMTS10_L14Q in the media and cell lysates.

\section{Immunofluorescence}

HEK 293E cells were grown on glass coverslips for $24 \mathrm{~h}$. After transient transfection with ADAMTS10-d2eGFP and ADAMTS10_L14Q-d2eGFP plasmid DNA, the cells were fixed with DPBS-buffered 3\% formaldehyde for $30 \mathrm{~min}$ and permeabilised with ice-cold methanol for $20 \mathrm{~s}$. For simultaneous staining of the endoplasmic reticulum (ER), rabbit anti-ERp72 (1:100, from Cell Signaling Technology) was used and visualised with Alexa 568 goat antirabbit antibodies (1:250, from Invitrogen). For nuclear staining, the cells were incubated with DAPI (1:1000 in DPBS) for $5 \mathrm{~min}$ and analysed by fluorescence microscopy (Zeiss, Oberkochen, Germany).

\section{Deglycosylation assay}

Following transient transfection with ADAMTS10-Myc-His6 or ADAMTS10_L14Q-Myc-His6 constructs, cells were harvested when cultures reached $90 \%$ confluence and dissolved in ice-cold $1 \times$ lysis reagent, as described above. The supernatant was collected, and the protein concentration was determined by a modified Bradford assay (Bio-Rad). As described elsewhere, ${ }^{16}$ $40 \mu \mathrm{g}$ of protein extract was treated with 1000 units of peptide $N$-glycosidase (PNGase F, New England Biolabs, Beverly, MA, USA). The reactions were stopped in sodium dodecyl sulphate sample buffer at $95^{\circ} \mathrm{C}$ for $5 \mathrm{~min}$. Finally $40 \mu \mathrm{g}$ of protein were loaded onto a $10 \%$ SDS-polyacrylamide gel electrophoresis using Prosieve $50 \mathrm{Gel}$ solution (Lonza) and Tris/tricine electrode buffer (0.1 M Tris, $0.1 \mathrm{M}$ Tricine, $0.1 \%$ SDS, $\mathrm{pH}$ 8.3) and electroblotted onto nitrocellulose membrane. Protein expression was assessed by western blotting analysis using a primary antibody directed against the Myc tag (monoclonal mouse anti-myc antibody 9E10 (1:100), from Invitrogen) and as a secondary antibody a horseradish peroxidase-labelled goat anti mouse antibody (SigmaAldrich) was used.

\section{RESULTS}

\section{Patient data}

An 18-year-old woman was initially referred to our Department for genetic evaluation of suspected atypical Noonan syndrome with pulmonic stenosis, short stature, and brachydactyly. The patient was born at term after an uneventful pregnancy from consanguineous parents (second-degree cousins, Supplementary Figure S2). After birth, a valvular pulmonary stenosis and a small muscular ventricular septal defect were observed. The VSD closed spontaneously. The valvular stenosis underwent an unsuccessful balloon catheterisation when the patient was 8 years old, with subsequent spontaneous regression. She developed a high degree of myopia $(-10 \mathrm{dpt})$ in early childhood, but no further ophthalmological abnormalities were reported.

Upon examination at age 19 years, the patient presented with short stature $(140 \mathrm{~cm})$, muscular build, scoliosis, and an accentuated lumbar lordosis. She was highly myopic ( $-13.5 \mathrm{dpt}$ OD and $-16 \mathrm{dpt}$ OS). She had joint stiffness at the elbows with reduced motility (supination $-23^{\circ}$ ) and was unable to make a fist with both hands. Her knees were also affected, and contracture of the calf musculature had led to an inability to stand with both feet parallel and simultaneously on the ground. She had marked brachydactyly. Her father had also brachydactyly and was of short stature, as well as her mother $(145 \mathrm{~cm})$. No other family members were affected.

The referral diagnosis of Noonan syndrome was based on her short stature and the presence of pulmonic stenosis. Brachydactyly was considered an independent familial dominant trait. Genetic analysis did not reveal functional variants in the PTPN11, SOS1, $K R A S$, and RAF1 genes. However, the pattern of brachydactyly, joint stiffness, ophthalmological symptoms, and heart defect led us to suspect a diagnosis of WMS, which was further strengthened by the finding of microspherophakia in a scheduled ophthalmological examination.

\section{Molecular genetics and in silico prediction}

We sequenced all exons of the ADAMTS10 gene in the patient and identified a homozygous nucleotide exchange, c.41T $>$ A. Both parents were heterozygous carriers. The variant is not listed in 1000 Genomes or in the exome variant browser. In silico mutation prediction analysis using Mutation Taster (http://www.mutationtaster.org) and PolyPhen-2 $2^{13}$ suggested that the variant was a benign variant. However, analysis with the SIFT program ${ }^{14}$ suggested that the variant affects function.

ADAMTS10 enters the secretory pathway during posttranslational modification. ${ }^{17}$ The base pair exchange described here affects the leucine at position 14, which is in the hydrophobic region of the leader peptide, resulting in an exchange to glutamine (p.(Leu14Gln)). Insight into the possible effect of an amino-acid exchange within a signal sequence can be gained with the SignalP signal peptide prediction program (http://www.cbs.dtu.dk/services/SignalP/). ${ }^{15}$ To assess whether the amino-acid exchange might result in protein mis-localisation because of a disrupted signal peptide, the $\mathrm{N}$-termini of the wild-type and mutant ADAMTS10 proteins were submitted to the SignalP V4.1 signal peptide prediction program. Analysis of the $60 \mathrm{~N}$-terminal amino acids of the ADAMTS10 wild-type protein predicted a cleavage site between amino-acid positions 25 and 26, with a $d$-score value of 0.759 for amino acids $1-25$, considerably exceeding the default $d$-score cutoff value of 0.450 (Figure 1a). In silico analysis of the c.41T $>$ A mutant, however, yielded a $d$-score value of only 0.446 for the first 25 amino acids, which are thereby no longer predicted to function as a signal peptide (Figure 1b).

\section{Disruption of the signal peptide leads to mis-localisation of ADAMTS10_L14Q}

To investigate whether the ADAMTS10 c.41T > A exchange leads to intracellular mis-localisation of the protein, plasmids containing eGFP fused to wild-type and ADAMTS10 c.41T > A cDNAs were transiently transfected in HEK 293E cells and examined by immunocytochemistry and fluorescence microscopy. In cells transfected with ADAMTS10d2eGFP, a considerable amount of the protein was present in the ER, as shown by co-visualisation with the ER marker ERp72 (Figure 2c). In contrast, ADAMTS10_L14Q-d2eGFP is localised to the cytosol in a 
a

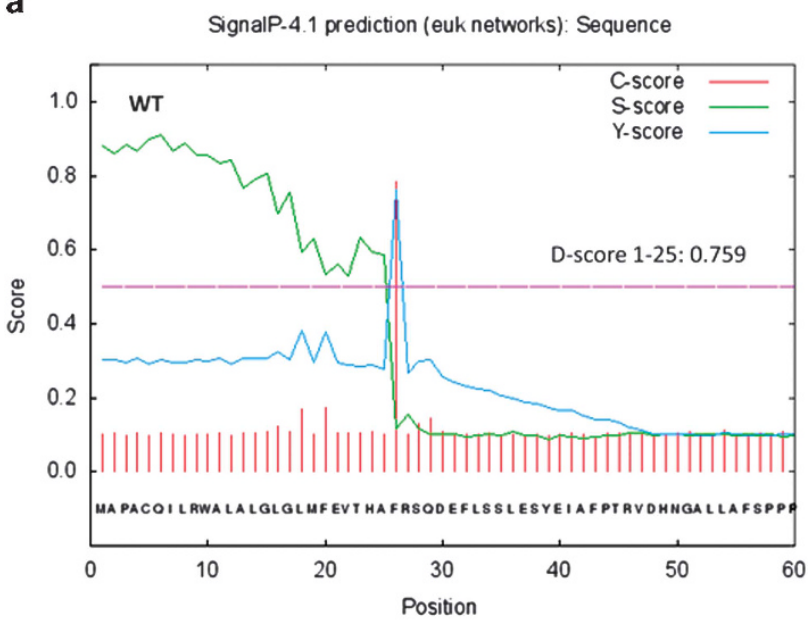

b

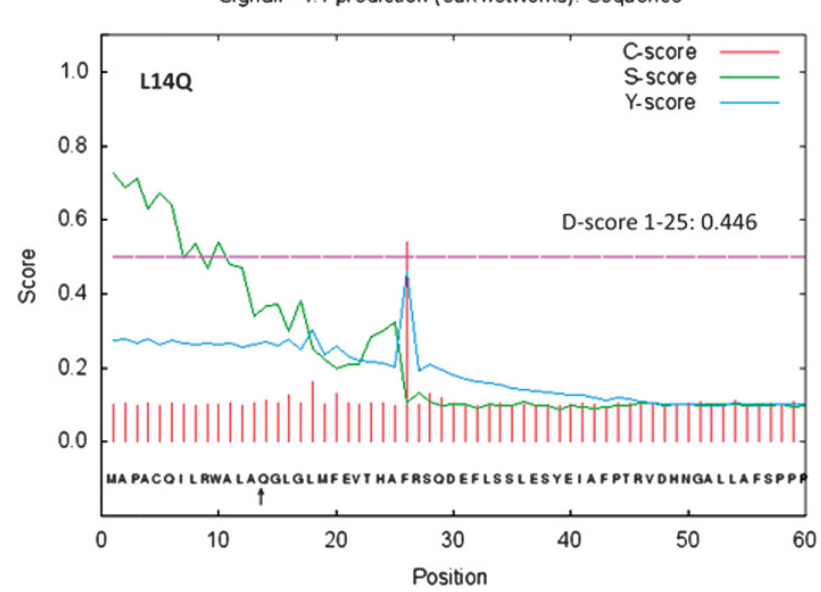

Figure 1 SignalP V4.1 graphic output for wild-type and mutant ADAMTS10 signal peptides. The $c$-score (raw cleavage site score) predicts the first amino-acid residue of the mature protein. The s-score (signal peptide score) calculates the probability of amino-acid residues to be part of a functioning signal peptide. The $y$-score combines the $c$-score and $s$-score, resulting in better cleavage site prediction. The $d$-score (discrimination score) is a weighted average of the mean s-score and the maximum $y$-score. (a) Analysis of the wild-type protein predicts a cleavage site between amino-acid positions 25 and 26 and yields a $d$-score value of 0.759 , well above the default cutoff for signal peptides. (b) The presence of a polar glutamine in exchange for leucine is predicted to disrupt the hydrophobic core of the ADAMTS10 signal peptide, thus reducing the probability that the amino-acid sequence acts as a signal peptide. The $d$-score value drops below the cutoff of 0.450

uniform diffuse pattern (Figure 2f). This finding confirms the in silico prediction and suggests that the leucine to glutamine exchange in the leader peptide leads to mis-targeting of the mutant protein to the cytoplasm. Next, we investigated the size of ADAMTS10 polypeptides by immunoblotting the media and cell lysates from HEK 293E cells transiently transfected with ADAMTS10-Myc-His6 and ADAMTS10_ L14Q-Myc-His6 constructs (Figure 3). In the cell lysates and supernatants of ADAMTS10-Myc-His6-transfected cells, we observed an anti-His6 reactive band at the expected size (approximately 140 $\mathrm{kDa})$. In cell lysates from ADAMTS10_LQ14-Myc-His6-transfected cells, a band at approximately $130 \mathrm{kDa}$ was observed, but no reactive band was present in the serum-free medium.
Impact of the $A D A M T S 10$ c.41T $>A$ variant on posttranslational modification

The different $\mathrm{kDa}$ values for the expressed ADAMTS10 fusion proteins prompted us to investigate whether the mobility shift is due to posttranslational modification. Protein extracts of HEK 293E cells transfected with the ADAMTS10-Myc-His6 and ADAMTS10_L14QMyc-His6 plasmid DNA were treated with endoglycosidase PNGase F (Figure 4). Endoglycosidase PNGase F cleaves $N$-acetylglucosamine side chains from asparagine residues and thus removes all asparagineassociated glycans. ${ }^{18}$ Treatment with PNGase F markedly increased the electrophoretic mobility of wild-type ADAMTS10 but had no effect on mutant ADAMTS10_L14Q, indicating that the mobility shift observed in untreated protein extracts is caused by a diminished $\mathrm{N}$-glycosylation of the mutated ADAMTS10 protein.

\section{DISCUSSION}

WMS is a very rare disease. In cases where the cardinal symptoms, such as microspherophakia, are not evident, this condition may be a clinically difficult diagnosis. In our case, at least two differential diagnoses were considered before a diagnosis of WMS was made. Because of the short stature, joint stiffness, and hyperlordosis, a hypoor achondroplasia was suggested. However, additional features, such as pulmonic stenosis and a missing rhizomelia, made this diagnosis unlikely. The association of short stature with micrognathia and pulmonary stenosis was also suggestive of Noonan syndrome, in which case the brachydactyly was considered a familial trait, due to the presence of brachydactyly in the father and, as reported by the patient, in other family members. This diagnosis was not confirmed by molecular analysis of genes that are commonly mutated in Noonan syndrome patients. Considering the co-occurrence of myopia, brachydactyly, and joint stiffness along with short stature, it became apparent that our patient might have a classic form of WMS. A new examination of her lens morphology strengthened this suspicion due to the identification of microspherophakia as the cause of the myopia in our patient. Subsequent variant analysis identified an unpublished missense variation in the ADAMTS10 gene. Using standard computer prediction programs (PolyPhen-2, Mutation Taster, and SIFT) for the analysis of unverified missense variants, no clear results could be obtained.

ADAMTS10 is a secreted protein with a signal peptide. Signal peptides co-translationally direct a nascent polypeptide chain from the cytosol to the ER and mediate the attachment of the ribosome to the ER membrane ${ }^{19}$ and the membrane insertion of newly synthesised secretory and membrane proteins. After fulfilling their function, signal peptides are cleaved from the precursor protein by a membranebound signal peptidase. ${ }^{20}$ Despite a great variation in both the overall length and amino-acid sequence, all export signal sequences contain a characteristic hydrophobic core (h-) region of 6-15 amino-acid residues for cleavable signal peptides. The sequence is flanked on its $\mathrm{N}$-terminal side by a polar (n-) region that usually has a net positive charge and on its $\mathrm{C}$-terminal side by a polar (c-) region containing the signal peptidase cleavage site, which is determined by small, uncharged residues at positions -1 and $-3 .^{21}$

Although SignalP V4.1 predicted amino acids $1-25$ of the wild-type ADAMTS10 protein to be a signal sequence, with a cleavage site located between Ala25 and Phe26, the substitution of a hydrophobic leucine with a hydrophilic polar glutamine within the centre of the hydrophobic core was suspected to have a detrimental effect on the function of the export signal peptide of ADAMTS10. In silico analysis thus supports the hypothesis that the reported variant c.41T $>\mathrm{A}$ has a detrimental effect on the ADAMTS10 signal sequence, possibly leading 

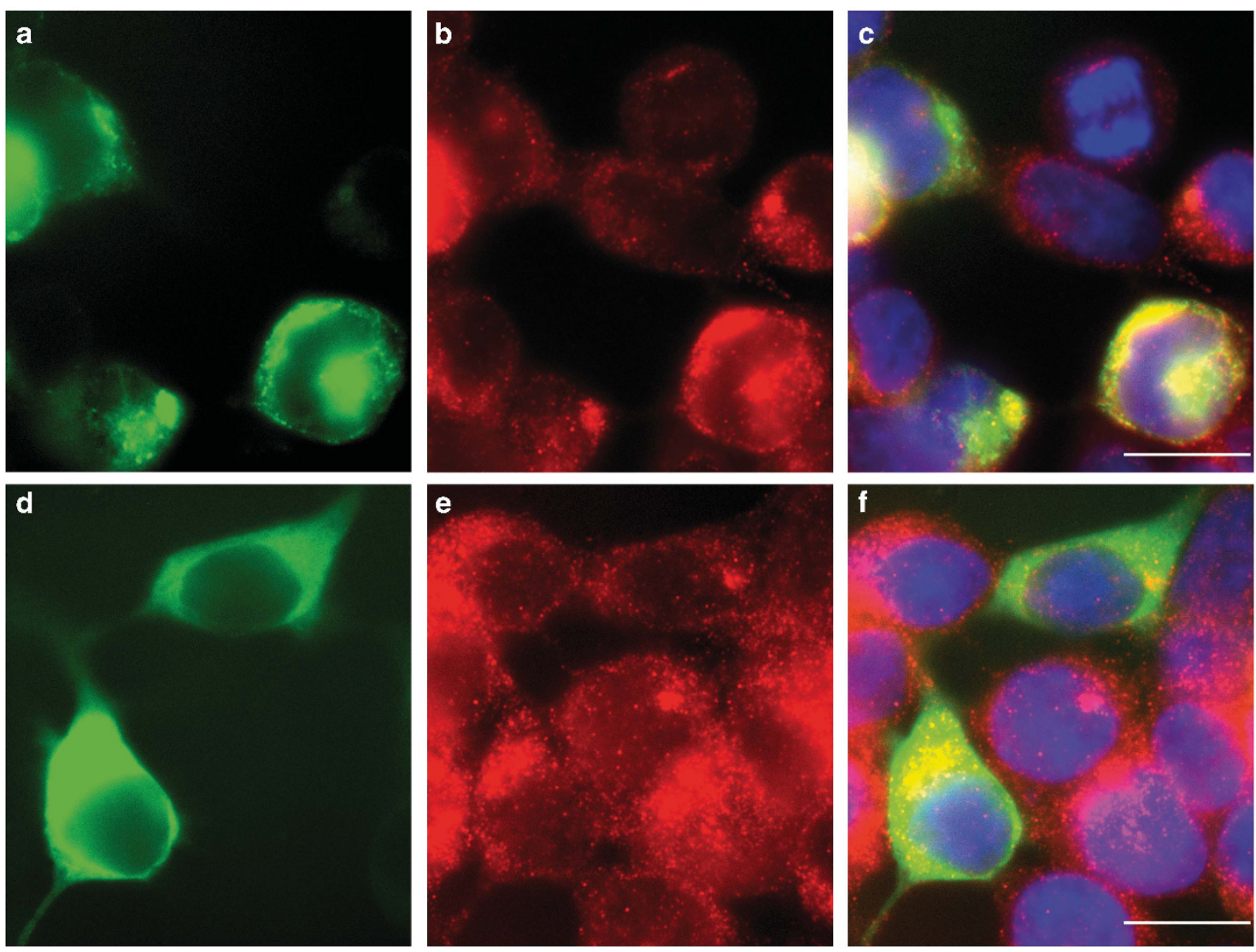

Figure 2 Localisation of ADAMTS10 and ADAMTS10_L14Q in HEK 293E cells. HEK 293E cells were transfected with ADAMTS10-d2eGFP (a-c) and ADAMTS10_L14Q-d2eGFP (d-f). To determine the localisation of ADAMTS10-d2eGFP and ADAMTS10_L14Q-d2eGFP, the cells were stained with the ER marker ERp72 (red). Cell nuclei were visualized by blue fluorescence (4,6-diamidino-2-phenylindole) in the merged images. The scale bar represents $10 \mu \mathrm{m}$. Most of ADAMTS10-eGFP protein is present in the ER (c), whereas ADAMTS10_L14Q-eGFP localises to the cytosol in a uniform, diffuse pattern (f).

a

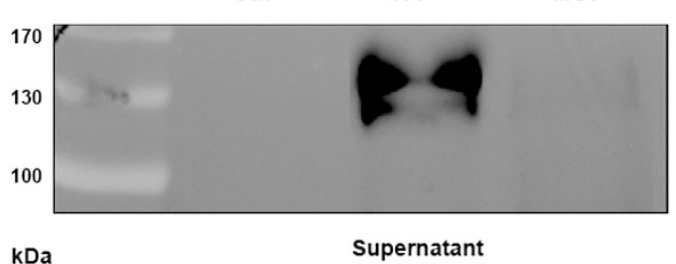

b

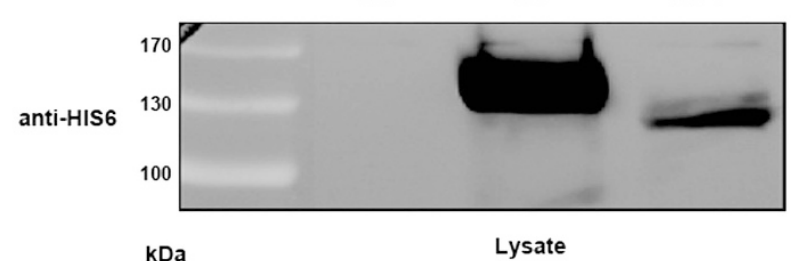

Figure 3 The expression pattern of ADAMTS10 and ADAMTS10-L14Q in HEK 293E cells. HEK 293 E cells were transfected with ADAMTS10-Myc-His6 and ADAMTS10_L14Q-Myc-His6 constructs. The polypeptides from supernatants (a) and cell lysates (b) were immunoblotted, and ADAMTS10 and ADAMTS10_L14Q protein detection in the cell lysate and the supernatant was performed using a polyclonal anti-His6 antibody. The ADAMTS10_L14Q protein was detected in the cell lysate as well as in the supernatant, whereas the ADAMTS10_L14Q polypeptide was only observed in the cell lysate, showing a mobility shift.

to impaired intracellular targeting. The hydrophobic core is required for targeting and membrane insertion. Most signal peptide variants affecting this region result in defective targeting to the ER and failed protein translocation. ${ }^{12,22-24}$

Therefore we expressed ADAMTS10-d2eGFP and ADAMTS10_ L14Q-d2eGFP proteins in HEK 293E cells and analysed their distribution by immunofluorescence staining for intracellular markers. Both wild-type and c.41T >A ADAMTS10 mRNA are readily translated into protein. The ADAMTS10-d2eGFP protein co-localised with the ER, but the ADAMTS10_L14Q-d2eGFP protein showed a more uniform, diffuse cytoplasmic distribution. Further analysis of the posttranslational processing revealed that in contrast to wild-type ADAMTS10, ADAMTS10_L14Q was not secreted into the extracellular matrix. Mis-targeting of the protein might also influence the posttranslational modification of ADAMTS10, as suggested by the reduced molecular weight of the intracellular ADAMTS10_L14Q protein. ADAMTS10 is a glycoprotein containing several N-linked glycosylation sites in various protein domains. ${ }^{17}$ Deglycosylation of wild-type ADAMTS10 and ADAMTS10_L14Q with endoglycosidase PNGase F revealed a mobility shift of the wild-type protein, indicating 


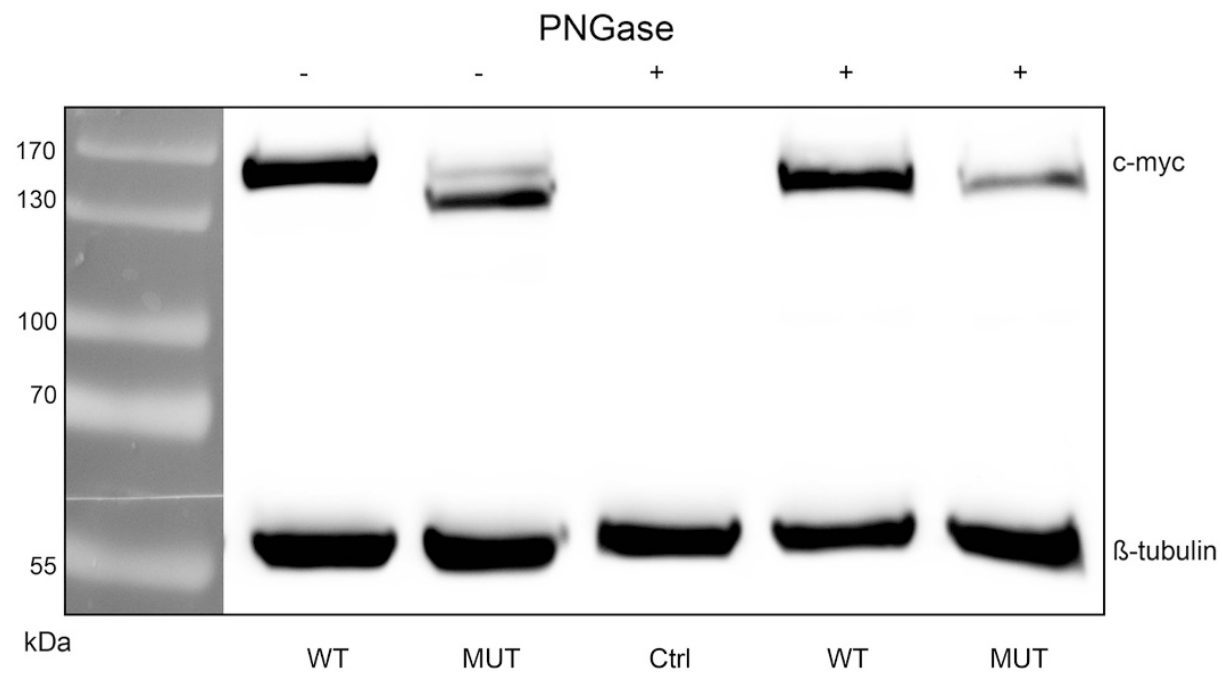

Figure $4 \mathrm{~N}$-linked deglycosylation assay of HEK 293E cells transfected with ADAMTS10-Myc-His6 or ADAMTS10_L14Q-Myc-His6. Whole cell extracts of HEK 293E cells transiently transfected with ADAMTS10-Myc-His6 (WT) and ADAMTS10_L14Q-Myc-His6 (MUT) or empty vector (Ctrl) were exposed to the PNGase $F(+)$ enzyme. The protein extracts were size-fractionated by sodium dodecyl sulfate-polyacrylamide gel electrophoresis under reducing conditions and characterised by western blotting analysis using an anti-c-myc antibody. The position of specific bands is indicated by a molecular mass marker (55$170 \mathrm{kDa}$ ), and $\beta$-tubulin detection was used to confirm uniform loading of the total protein.Whereas deglycosylation of the ADAMTS10_L14Q-Myc-His6 protein with PNGaseF does not alter protein mobility, PNGase treatment of ADAMTS10-Myc-His6 results in a mobility shift with an electrophoretic mobility corresponding to the ADAMTS10_L14Q-Myc-His6 protein.

defective glycosylation of the ADAMTS10_L14Q protein. Moreover, we found that ADAMTS10_L14Q exchange leads to protein instability in HEK 293E cells by cycloheximide translation inhibition experiments (see Supplementary Figure S1).

Although a missense variant at position -1 from the ADAMTS10 signal peptide cleavage site (p.Ala25Thr) has been described, ${ }^{11}$ this is the first report of an ADAMTS10 variant disrupting the hydrophobic core of the export signal sequence. Based on our results, we propose that the missense substitution leads to mis-targeting of the protein. The mutant RNA is translated, but eGFP-tagged ADAMTS10_L14Q could not be observed in the ER. Mutant ADAMTS10_L14Q protein translation might take place in free ribosomes instead, with cytosolic deposition and no secretion of the protein. $\mathrm{N}$-glycosylation takes place co-translationally in the ER, and mis-targeting therefore inhibits effective glycosylation of ADAMTS10_L14Q. Extracellular secretion of ADAMTS10 is essential for its function in the extracellular matrix. The altered cytosolic unglycosylated ADAMTS10 cannot act as wildtype ADAMTS10, resulting in the WMS phenotype of the patient. Taken together, our findings suggest that the exchange of a leucine for a hydrophilic polar glutamine within the hydrophobic core disrupts the function of the ADAMTS10 signal peptide, thereby impairing correct intracellular trafficking and co-translational glycosylation of the protein. The ADAMTS10 mutated protein is secretion-deficient. We conclude that the homozygous ADAMTS10 c.41T > A missense variant reported here is causative for WMS.

\section{CONFLICT OF INTEREST}

The authors declare no conflict of interest.

\section{ACKNOWLEDGEMENTS}

We thank Clemens Röhrl for technical assistance with immunofluorescence microscopy; Matthias Spork for scientific input; and Markus Galhuber, Britta Kluge, and Manuela Kropik for technical assistance.

\section{AUTHOR CONTRIBUTIONS}

HS, JE, and LG performed the experiments. Clinical studies are from ES, OB, and FL. HS, JE, JN, OB, and FL designed the study and wrote the manuscript.

1 Faivre L, Dollfus $\mathrm{H}$, Lyonnet $\mathrm{S}$ et al: Clinical homogeneity and genetic heterogeneity in Weill-Marchesani syndrome. Am J Med Genet A 2003; 123A: 204-207.

2 Sengle G, Tsutsui K, Keene DR et al: Microenvironmental regulation by fibrillin-1. PLoS Genet 2012; 8: e1002425.

3 Paton D, Murdoch JL: Weill-Marchesani syndrome. Birth Defects Original Article Series 1971; 7: 174-177.

4 Jensen AD, Cross HE, Paton D: Ocular complications in the Weill-Marchesani syndrome. Am J Ophthalmol 1974; 77: 261-269.

5 Wright KW, Chrousos GA: Weill-Marchesani syndrome with bilateral angle-closure glaucoma. J Pediatr Ophthalmol Strabismus 1985; 22: 129-132.

6 Dagoneau N, Benoist-Lasselin C, Huber C et al: ADAMTS10 mutations in autosomal recessive Weill-Marchesani syndrome. Am J Hum Genet 2004; 75: 801-806.

7 Young ID, Fielder AR, Casey TA: Weill-Marchesani syndrome in mother and son. Clin Genet 1986; 30: 475-480.

8 Haji-Seyed-Javadi R, Jelodari-Mamaghani S, Paylakhi SH et al: LTBP2 mutations cause Weill-Marchesani and Weill-Marchesani-like syndrome and affect disruptions in the extracellular matrix. Hum Mutat 2012; 33: 1182-1187.

9 Cain SA, McGovern A, Baldwin AK, Baldock C, Kielty CM: Fibrillin-1 mutations causing Weill-Marchesani syndrome and acromicric and geleophysic dysplasias disrupt heparan sulfate interactions. PLoS One 2012; 7: e48634.

10 Cecchi A, Ogawa N, Martinez HR et al: Missense mutations in FBN1 exons 41 and 42 cause Weill-Marchesani syndrome with thoracic aortic disease and Marfan syndrome. Am J Med Genet A 2013; 161: 2305-2310.

11 Morales J, Al-Sharif L, Khalil DS et al: Homozygous mutations in ADAMTS10 and ADAMTS17 cause lenticular myopia, ectopia lentis, glaucoma, spherophakia, and short stature. Am J Hum Genet 2009; 85: 558-568.

12 Kutz WE, Wang LW, Dagoneau N et al: Functional analysis of an ADAMTS10 signal peptide mutation in Weill-Marchesani syndrome demonstrates a longrange effect on secretion of the full-length enzyme. Hum Mutat 2008; 29: 1425-1434.

13 Adzhubei IA, Schmidt S, Peshkin L et al: A method and server for predicting damaging missense mutations. Nat Methods 2010; 7: 248-249.

14 Kumar P, Henikoff S, Ng PC: Predicting the effects of coding non-synonymous variants on protein function using the SIFT algorithm. Nat Protoc 2009; 4: 1073-1081.

15 Petersen TN, Brunak S, von Heijne G, Nielsen H: SignalP 4.0: discriminating signal peptides from transmembrane regions. Nat Methods 2011; 8: 785-786.

16 Lu C, Walker WH, Sun J et al: Insulin-like peptide 6: characterization of secretory status and posttranslational modifications. Endocrinology 2006; 147: 5611-5623. 
17 Somerville RP, Jungers KA, Apte SS: Discovery and characterization of a novel, widely expressed metalloprotease, ADAMTS10, and its proteolytic activation. J Biol Chem 2004; 279: 51208-51217.

18 Maley F, Trimble RB, Tarentino AL, Plummer TH Jr: Characterization of glycoproteins and their associated oligosaccharides through the use of endoglycosidases. Anal Biochem 1989; 180: 195-204.

19 Walter $\mathrm{P}$, Johnson $\mathrm{AE}$ : Signal sequence recognition and protein targeting to the endoplasmic reticulum membrane. Annu Rev Cell Biol 1994; 10: 87-119.

20 von Heijne G: Life and death of a signal peptide. Nature 1998; 396: 111, 113.

21 Martoglio B, Dobberstein B: Signal sequences: more than just greasy peptides. Trends Cell Biol 1998; 8: 410-415.
22 Hughes AE, Ralston SH, Marken J et al: Mutations in TNFRSF11A, affecting the signal peptide of RANK, cause familial expansile osteolysis. Nat Genet 2000; 24: 45-48.

23 Seppen J, Steenken E, Lindhout D, Bosma PJ, Elferink RP: A mutation which disrupts the hydrophobic core of the signal peptide of bilirubin UDP-glucuronosyltransferase, an endoplasmic reticulum membrane protein, causes Crigler-Najjar type II. FEBS Lett 1996; 390: 294-298.

24 Pidasheva S, Canaff L, Simonds WF, Marx SJ, Hendy GN: Impaired cotranslational processing of the calcium-sensing receptor due to signal peptide missense mutations in familial hypocalciuric hypercalcemia. Hum Mol Genet 2005; 14: 1679-1690.

Supplementary Information accompanies this paper on European Journal of Human Genetics website (http://www.nature.com/ejhg) 